VIII ${ }^{\text {èmes }}$ Journées Nationales Génie Civil - Génie Côtier, Compiègne, 7-9 septembre 2004

\title{
Protection du chenal d'accès à la marina Puerto Bonito en République Dominicaine
}

\author{
Jean-Marc Beynet $^{(\mathrm{a})}$, Jean Bougis ${ }^{(\mathrm{b})}$, François Carnus $^{(\mathrm{c})}$, Pierre Fehlmann ${ }^{(\mathrm{d})}$ \\ ${ }^{(a)}$ Directeur de projet, BRL Ingénierie, 1105 Avenue P. Mendès France, 30001 Nîmes Cedex, \\ France, Tél. : 33 (0) 4.66.87.51.14 jean-marc.beynet@brl.fr \\ ${ }^{(b)}$ Ingénieur Conseil, 34, Chemin du Moulin, 06650 Opio, France \\ Tél. : 33 (0) 4.93.77.74.22, jbougis@aws.fr \\ ${ }^{(c)}$ Expert en environnement maritime, BRL ingénierie, 1105, Avenue Pierre Mendès France \\ 30001 Nîmes Cedex, Tél : 33 (0) 4.66.87.50.68 françois.carnus@brl.fr \\ ${ }^{(d)}$ Président de la Société marina Puerto Bonito SA, PO Box 1631110 Morges, \\ Switzerland. Tél : 41 (0) 2180322 01, pierrefehlmann@compuserve.com
}

\section{Résumé:}

Cet article présente les études menées pour concevoir le chenal d'accès à la marina Puerto Bonito proche de Las Terrenas en République Dominicaine. Pour minimiser les impacts dans une baie au littoral vierge de toute construction, le promoteur et les concepteurs ont pris en compte les différentes problématiques posées par le choix de la protection du chenal d'entrée sur le plan fonctionnel de la navigation, sur le plan de la réalisation des ouvrages de génie civil, ainsi que sur le plan environnemental (aspects hydrodynamiques et sédimentologiques).

\begin{abstract}
:
This paper describes the design studies of the access channel to the marina Puerto Bonito, located near Las Terrenas in Dominicain Republic. In order to minimize impacts in a bay where the littoral is virgin of any structures and buildings, the owner and the designers have taken into consideration different problems as protection, navigation, erection of civil works structures and environmental aspects (hydrodynamic and sedimentologic studies).
\end{abstract}

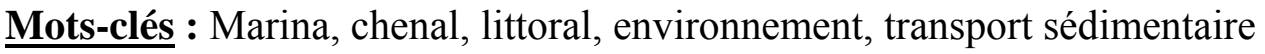

Keywords: Marina, channel, littoral, environment, sediment transport

\section{Introduction}

La République Dominicaine étend son territoire sur les deux tiers orientaux de l'île d'Hispagnola qu'elle partage avec la République d'Haïti. Dotée d'un patrimoine naturel exceptionnel, elle conduit une politique volontariste pour le développement du tourisme dans le plus grand respect de son environnement. La création de la Marina Puerto Bonito, près de Las Terrenas, dans une baie au littoral vierge de toute construction, s'inscrit dans le projet l'aménagement du littoral de la côte Nord-Est de l'île. La Marina Puerto Bonito étant destinée à l'accueil des bateaux de grande plaisance, jusqu'à une longueur de $50 \mathrm{~m}$ et un tirant d'eau de 4,0 $\mathrm{m}$, son accès maritime nécessite l'aménagement d'un chenal d'une profondeur d'eau minimale de 4,5 m. Ce chenal s'ouvrira au Nord-Ouest, dans la partie Est de la baie Escocesa, juste à l'Est de la Punta Bonita. Cette portion du littoral est protégée à l'Est par la Punta Bobilanza. Les fonds marins descendent rapidement pour atteindre $500 \mathrm{~m}$ de profondeur à environ trois milles nautiques du rivage. Afin de préserver la qualité de l'environnement et l'aspect naturel du littoral, la marina sera entièrement construite autour de bassins creusés à l'intérieur des terres et ne sera visible de la mer que par le débouché de son chenal d'accès. 


\section{$\underline{\text { 2.Etudes hydrodynamiques et sédimentologiques }}$}

Pour orienter les choix concernant l'implantation et les dispositions constructives du chenal, il est nécessaire de maîtriser les données naturelles du site et en particulier les contraintes hydrodynamiques et sédimentologiques qui ont une importance particulière puisque l'île d'Hispagnola est située en zone cyclonique.

\subsection{Données bathymétriques et niveaux de référence}

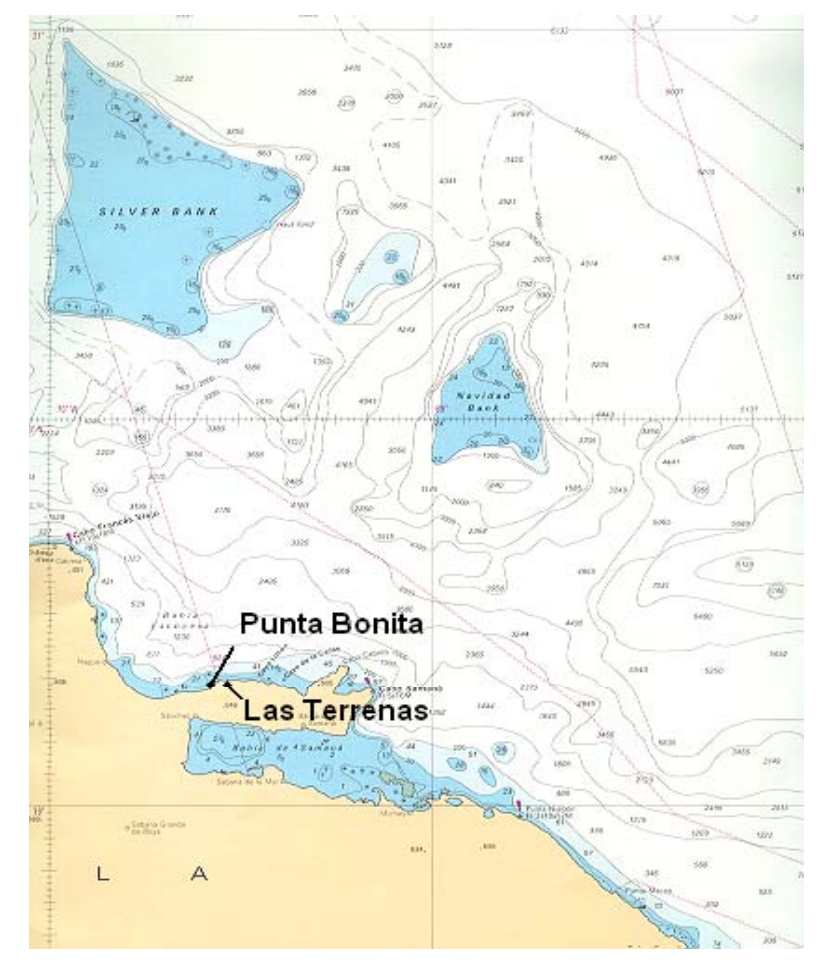

Figure 1: Bathymétrie au large du site

Extrait en réduction de la carte SHOM N7474 au 1/1 000000

Les données bathymétriques sont issues de différentes sources (cartes marines françaises, britanniques et américaines, cartes terrestres locales) complétées par des levés bathymétriques et topographiques réalisés dans le cadre du projet. Chaque type de document possède son propre système de coordonnées et de niveau de référence. Les cotes ont donc été réduites au zéro hydrographique des cartes françaises, très proche du nivellement générale (NGRD). Il existe le long des côtes d'Hispagnola des zones incomplètement hydrographiées.

Les niveaux des marées astronomiques et météorologiques résultent de la synthèse de documents pas toujours très cohérents. Le niveau moyen de la mer s'établi à $+0.25 \mathrm{~m} \mathrm{CM}$ avec des variations extrêmes de $-0.30 \mathrm{~m}$ à $+0.80 \mathrm{~m}$. En dehors des conditions cycloniques, les marées barométriques ont une amplitude de $\pm 0.2 \mathrm{~m}$, tandis, que sur le site assez ouvert de la Playa Bonita, les marées atmosphériques cycloniques atteignent 1.0 à $1.5 \mathrm{~m}$. Le niveau maximal de la mer est de $1.0 \mathrm{~m} \mathrm{CM}$ en régime normal, et de $2.0 \mathrm{~m}$ sous les cyclones.

\subsection{Climats de houles}

Au large, les hauts fonds (Navidad Bank et Sylver Bank) susceptibles de déformer les champs de houles incidents, imposent l'utilisation d'un modèle d'approche pour transférer à la côte les houles du large définies à partir de données statistiques britanniques. Ce modèle couvre les fonds inférieurs à $1000 \mathrm{~m}$, au delà des hauts fonds au Nord et de Puerto Rico à l'Est 
(425 km Nord-Sud et $525 \mathrm{~km}$ Est-Ouest). Lors de leur passage sur ces hauts fonds, les houles longues sont puissamment freinées par le raccourcissement de leur longueur d'onde. De part et d'autre, les deux nappes extérieures, plus rapides, tournent sous l'effet de la réfraction et de la diffraction générées par les tombants et viennent se croiser. Ces focalisations engendrent des caustiques qui concentrent l'énergie dans un secteur angulaire très étroit. Le plateau étant assez large, dès que la profondeur relative est suffisante importante, la nappe principale sort des hauts fonds et reprend sa course, droit devant elle, avec des crêtes bien parallèles. Elle véhicule une énergie demeurée importante avec peu de dispersion spatiale. Par contre, les nappes latérales s'épanouissent sous l'effet de la diffraction et leur énergie se disperse dans l'espace. Ainsi, selon les longueurs, une (houles courtes), deux (houles longues), ou trois (houles moyennes) nappes de houles sont dissociées par les hauts fonds.

A l'arrivée sur le site de la Playa Bonita, toutes les nappes sont réfractées par le plateau limité par les lignes bathymétriques de 20 à $30 \mathrm{~m}$ et se présentent avec une incidence de secteur Nord à quelques degrés près. Devant le site, les hauteurs des nappes secondaires sont de l'ordre de 20 à $30 \%$ de la hauteur de la nappe principale pour les périodes moyennes et sont négligeables pour les périodes courtes. Il est très difficile de distinguer les différentes nappes qui arrivent sur le site à la même période dans un faisceau d'incidence très étroit. Elles donnent l'impression de houles plus courtes et plus hachées. Leur période étant quasiinchangée, le risque d'excitation de seiches basses fréquences n'est pas augmenté par rapport à une houle qui arriverait en une seule nappe. Le tableau 1 présente les spectres de houles locaux qui ont été retenus pour les études d'agitation des abords de la marina.

Tableau 1: Spectres des états de mer caractéristiques de la Playa Bonita

\begin{tabular}{|c|c|c|c|c|c|c|c|c|}
\hline Incidence & $\mathrm{N} 350^{\circ}$ & $\mathrm{N} 350^{\circ}$ & $\mathrm{N} 355^{\circ}$ & $\mathrm{N} 355^{\circ}$ & $\mathrm{N} 0^{\circ}$ & $\mathrm{N} 0^{\circ}$ & $\mathrm{N} 5^{\circ}$ & $\mathrm{N} 10^{\circ}$ \\
$H_{\mathrm{s}}(\mathrm{m})$ & 1.20 & 1.70 & 1.40 & 1.20 & 1.35 & 1.35 & 0.65 & 0.40 \\
$T_{\mathrm{p}}(\mathrm{s})$ & 7.0 & 9.0 & 7.5 & 9.5 & 8.5 & 10.0 & 9.5 & 9.0 \\
\hline
\end{tabular}

\subsection{Contexte sédimentologique}

Comme le montre la photographie aérienne (Figure 5 ci-après), isolée entre deux caps rocheux, la baie de la Playa Bonita n'échange quasiment pas de matériaux solides avec les portions de côte adjacentes. A l'Est, la pointe rocheuse offre une bonne résistance à l'érosion. A l'Ouest, bien que protégée par un platier corallien qui fait déferler les vagues à quelques centaines de mètres du rivage, la Punta Bonita plus meuble est en forte érosion; de un à deux mètres par an. La plage sablonneuse étroite est adossée à la côte concave. Au Nord-Est, à proximité de l'embouchure d'un petit rio, la plage est interrompue par deux éperons rocheux, créant ainsi deux petites plages apparemment isolées de la plage principale.

En plusieurs endroits, la présence d'un "beach rock" témoigne de l'attaque frontale des houles : les mouvements sédimentaires se font essentiellement dans le profil. Le haut de la plage de Playa Bonita s'étend sur une largeur variable de quelques mètres à quelques dizaines de mètres selon les endroits, avec une pente franchement plus forte que celle de la plage. Il se termine par une dune basse régulière qui décroît d'Ouest en Est. A l'Est, le front dunaire est formé de microfalaises d'érosion plus ou moins stabilisées par la végétation. La route du bord de mer est implantée sur l'arrière du cordon dunaire. La dune est stabilisée sur la totalité de la longueur de la baie par une végétation de cocotiers, complétée en certains endroits par une végétation basse composée d'herbes et de plantes. Cette stabilisation empêche tout échange du cordon dunaire avec la zone terrestre. 
La granulométrie décroît d'Ouest en Est depuis la Punta Bonita jusqu'au rio. C'est le contraire qui se produit au Nord-Est du rio, mais avec un gradient beaucoup plus faible. Cela traduit que l'embouchure du rio est située au point de transit longitudinal nul, ce qui est naturel puisque le transit tend à boucher le rio et à le déplacer dans son sens. Sauf à avoir un fort débit et/ou une topographie marquée qui imposent la position de l'exutoire, le rio ne peut guère se forger un passage qu'en ce lieu. Ce point est d'ailleurs bien situé par rapport à la topographie vis-à-vis de l'écoulement naturel des eaux.

Les mouvements sédimentaires dans la baie obéissent à deux régimes de fonctionnement:

- Un transit sédimentaire des sables moyens, principalement par charriage et saltation, engendré par les courants de houle le long de la plage d'Ouest en Est depuis la Punta Bonita jusqu'à l'embouchure du rio et du Nord au Sud de l'autre côté du rio. L'obliquité des houles étant faible, ce transit est assez lent.

- Un transit sédimentaire de sable fin, principalement par suspension, engendré par les courants de houles qui prennent naissance sur les hauts fonds au large de la punta Bonita et qui se dirige vers la limite Nord-Est de la baie de la Playa Bonita. Il alimente les plages situées au Nord du rio et le transit littoral du Nord vers le Sud dont elles sont le siège.

Il résulte de ces cheminements que le sable fin alimente les deux plages du Nord-Est qui ne sont donc pas en équilibre autarcique et participent à l'équilibre de l'ensemble de la baie. Ce phénomène explique que ces plages ne démaigrissent pas malgré un transit du Nord vers le Sud comme en témoignent les analyses granulométriques et les accumulations de sable.

\section{Optimisation du tracé du chenal d'accès}

Les contraintes prises en compte pour l'optimisation du chenal d'accès sont :

- La préservation de la stabilité du littoral et de l'aspect naturel du site,

- La navigation et manoeuvrabilité,

- La signalisation et le balisage,

- Les conditions d'agitation du chenal et de l'entrée de la marina,

- Les risques d'ensablement du chenal et de l'entrée de la marina,

- Le renouvellement des eaux de la marina,

- Les coûts de création et d'entretien du chenal.

\subsection{Stabilité du littoral et préservation de l'aspect naturel du site}

Pour conserver la stabilité du littoral, il est indispensable de laisser passer les courants générés au large de la Punta Bonita pour qu'ils puissent nourrir en sédiments les plages du Nord-Est. L'interruption de cet apport, alors même que les atteintes de la houle seraient aussi diminuées, engendrerait sans doute à terme la disparition des plages et le sable apporté par les courants se déposerait le long de la digue. L'équilibre et la stabilité hydrosédimentaire globale de la baie seraient alors menacés. Par ailleurs, les différentes données physiques d'origines géographique, hydrodynamique et sédimentologique, conduisent à chercher à enraciner le chenal d'accès au point de transit nul. 


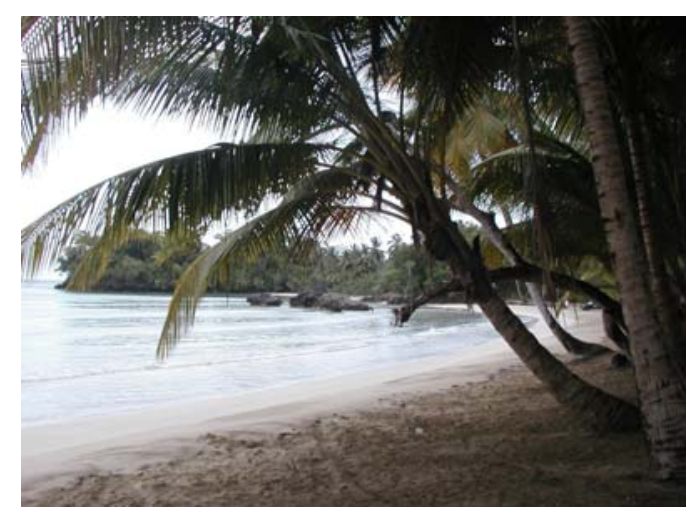

Figure 2: Playa Bonita

Le site de Playa Bonita est particulièrement beau et il est resté complètement naturel. Le respect de ce site et la préservation de son apparence et de sa qualité s'impose pour ne pas dénaturer le paysage paradisiaque de la baie. Pour ne pas couper la vue et limiter les impacts visuels des ouvrages d'accès à la marina, le chenal sera bordé par des digues submergées destinées à limiter l'ensablement. Les digues n'émergeront qu'à leur enracinement et le long du premier tronçon de chenal (voir tracé du chenal et profils-en travers types sur les Figures 3 et 4 ci-après). Leur hauteur sera limitée au strict nécessaire. Au-delà, le transit littoral dû aux courants de lévigations devient quasiment inexistant.

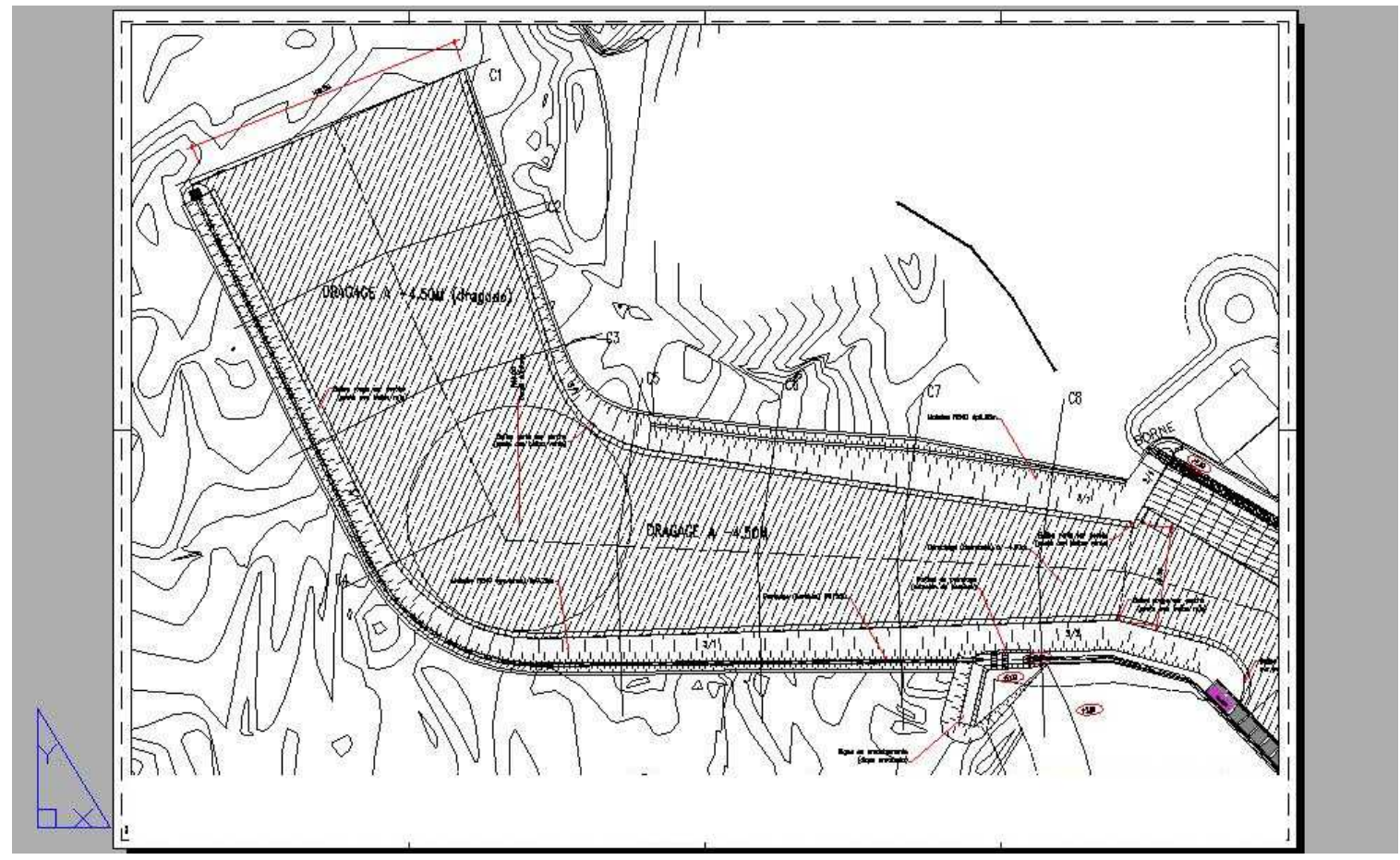

Figure 3: vue en plan du chenal 


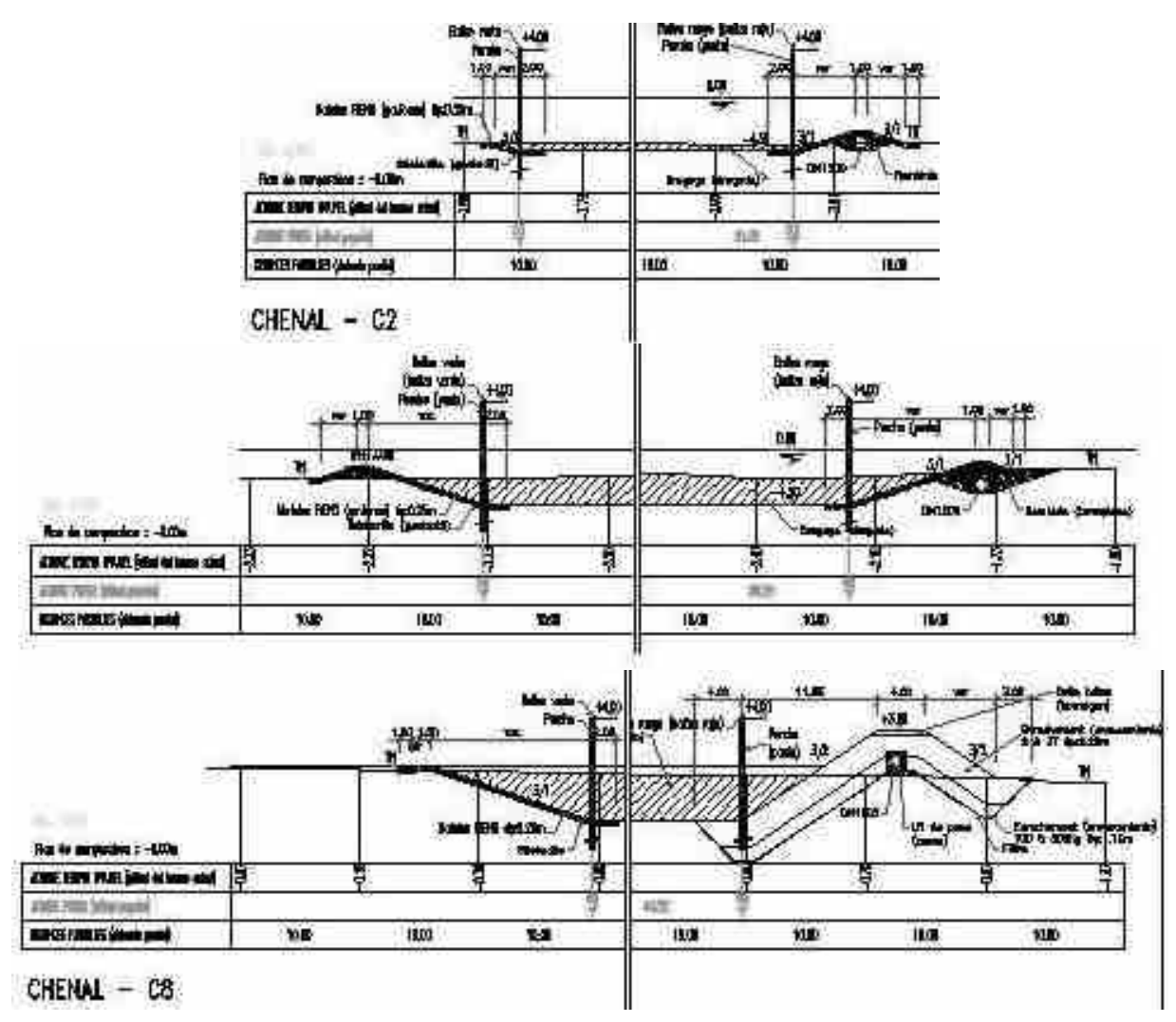

Figure 4: profils en travers types C2, C6, C8

\subsection{Navigation et manoeuvrabilité des navires}

La cote de dragage d'un chenal est fonction du tirant d'eau des navires qui y transitent et d'un "pied" de pilote qui tient compte :

- Du surenfoncement du navire lié à sa vitesse et à la forme de sa carène. Pour des voiliers navigant à 2 ou 3 noeuds, Il reste inférieur à $5 \mathrm{~cm}$.

- De la dépression moyenne de dérive sur houle. Pour les mêmes navires, la vitesse orbitale d'une houle de $H=1 \mathrm{~m}$ et $T=6 \mathrm{~s}$, est au plus de $1 \mathrm{~cm}$.

- Des mouvements des navires sur houle. Ces navires ont des périodes propres de pilonnement et de tangage de 2 à 4 secondes. Au-delà de ces périodes, les amplitudes des mouvements verticaux sont inférieures à celle de la houle et le navire descendra au plus de $H / 2$ au dessous du niveau moyen de l'eau.

Un pied de pilote de $0.5 \mathrm{~m}$ sera suffisant si la hauteur de la houle dans le chenal est inférieure à $1.0 \mathrm{~m}$; compte tenu du marnage $(-0.3 \mathrm{~m}$ à $+0.8 \mathrm{~m})$, une profondeur de $4.5 \mathrm{~m} \mathrm{CM}$ sera alors suffisante pour le chenal, sous réserve des variations dues aux marées astronomiques et météorologiques pour les navires à fort tirant d'eau. 


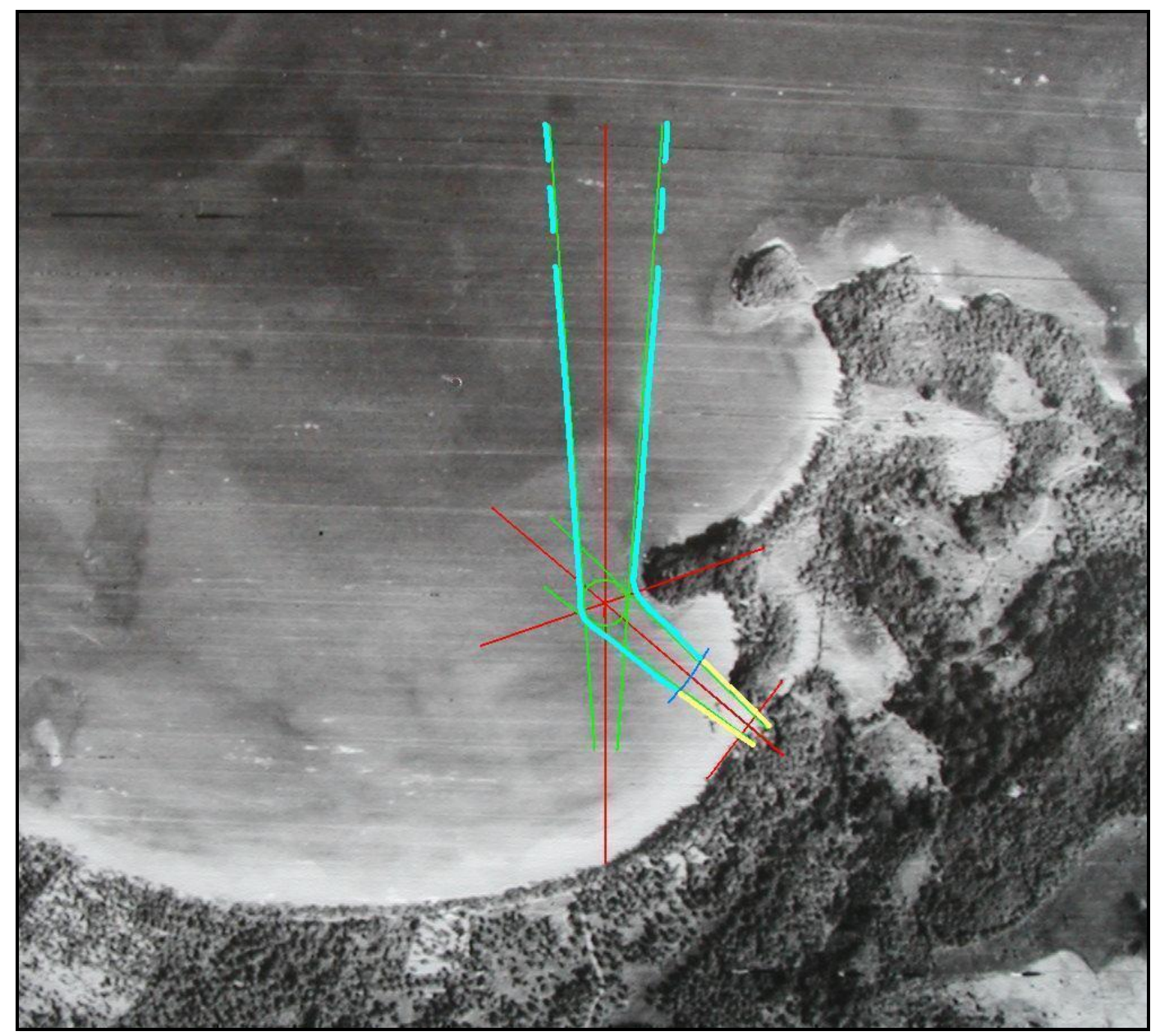

Figure 5: Tracé du chenal d'accès à la Marina Puerto Bonito

Les vents généraux soufflent essentiellement du secteur Nord-Ouest à Nord-Est. Sauf lorsque les vents thermiques nocturnes de terre les masquent, les navires auront le vent arrière pour entrer dans la marina et le vent de face pour en sortir. Il sera difficile à un navire de fort tirant d'eau de sortir du chenal à la voile, mais il sera toujours possible à tous les navires d'y entrer. Pour des navires de $20 \mathrm{~m}$ à $25 \mathrm{~m}$ de long, une largeur du chenal de $50 \mathrm{~m}$ à $70 \mathrm{~m}$ sera suffisante. Il s'élargira progressivement à $100 \mathrm{~m}$ pour faciliter la manœuvre depuis le large. Pour limiter la distance sur laquelle les navires sont peu manoeuvrants, le tracé le plus court a été recherché en rejoignant la ligne de sondes des $5 \mathrm{~m} \mathrm{CM}$ le plus directement possible. La bathymétrie du site incite donc à rechercher un tracé direct vers le Nord.

\subsection{Signalisation et balisage nautique}

Outre le balisage latéral indispensable, matérialisé par des perches à l'Est et à l'Ouest, il est préférable de constituer le chenal de deux ou trois tronçons rectilignes pour pouvoir le matérialiser par des alignements ou des feux à secteurs.

\subsection{Agitation du chenal et de la passe d'accès à la marina}

Les houles dominantes sur le site sont de secteur Nord-Nord-Ouest à Nord et le chenal sera agité par la plupart des houles. Il convient d'éviter que les navires soient pris par la houle par le trois-quarts arrière, situation qui engendre plus de difficultés (mouvements d'embardée et de lacet, stabilité de route) que la houle d'arrière. Là encore un tracé le plus au Nord possible semble la meilleure solution. 


\subsection{Ensablement du chenal et de l'entrée de la marina}

L'ouverture de la marina au point de transit littoral nul, est de nature à en limiter l'ensablement par le transit dû au jet de rive. Pour tirer au mieux profit de cet état des choses, il faut rechercher un tracé qui soit normal au trait de côte en ce point. Cela est d'ailleurs conforme à la nécessité de suivre la ligne de plus grande pente pour limiter les volumes de dragage lors de la création du chenal.

\subsection{Renouvellement des eaux de la marina}

Dans ce site à très faible marnage, le renouvellement des eaux de la marina doit être assuré par une circulation artificielle qui prélève l'eau à l'extrémité du chenal et la refoule en divers points en fond de darse. Cet apport induit un courant de vidange par le chenal d'entrée. Le circuit inverse ne permettrait pas de contrôler la qualité de l'eau entrante. En termes de "pollution" des eaux, le chenal joue, dans son premier tronçon, le rôle d'un "émissaire" de rejet. Il doit donc permette la meilleure dilution possible des eaux sortantes. Ce qui a deux conséquences :

- Le point de départ doit être situé dans une zone où le courant part vers le large ; c'est le cas du point de rencontre des courants de houle venant des deux côtés.

- Le chenal doit suivre la plus grande pente de la plage.

\subsection{Coûts de création et d'entretien du chenal}

Les coûts de création du chenal sont liés au volume et à la nature des fonds à draguer. Pour les limiter, il faut suivre la ligne de plus grande pente de la plage et aller au plus court vers les fonds navigables. Pour limiter les coûts d'entretien il faut que le chenal s'ensable le moins possible et minimiser la surface à draguer.

\section{Proposition de tracé}

Compte tenu des contraintes analysées ci-dessus, le tracé du chenal peut être établi. La figure 5 représente le tracé du chenal avec ses axes, et ses traits de construction. Seul le premier tronçon du chenal est bordé de digues émergées. Le segment de départ du chenal est normal au trait de côte pour empêcher les houles d'entrer dans la marina, et protéger au mieux l'alimentation en sédiments de la première plage située au Nord de l'embouchure du rio. Les parties de digues émergées ne doivent pas être trop longues pour ne pas capter les courants générés par les déferlements sur les platiers coralliens au large de la Punta Bonita.

\section{Proposition de profil en travers}

Les coupes en travers des digues émergées ne posent pas de problème particulier puisque les hauteurs de la houle sur site sont faibles et ne nécessitent pas des blocs très gros. Le profil des digues immergées est plus délicat à établir. Leur cote d'arase au dessus du sol doit permettre d'opposer un obstacle suffisamment haut au transport sédimentaire par charriage et saltation, sans couper les courants. Un compromis a été adopté en les arasant entre 0.5 et 1.0 $\mathrm{m}$ au dessus du sol naturel de l'entrée du chenal côté large jusqu'au cercle de jonction entre les deux tronçons du chenal. Au delà, la digue Nord est émergée, tandis que la digue Sud peut soit être continuée ainsi jusqu'au point où elle émerge, en créant une discontinuité, soit être raccordée à la côte d'arase de la partie émergée dès la sortie du cercle de jonction, sans discontinuité. Ces discontinuités sont traitées comme des musoirs, avec des enrochements plus gros. Les pentes des digues immergées sont assez douces (2/1 à 3/1). Des risbermes sont prévues en pied du côté du chenal. Les digues émergées sont plus raides avec des enrochements de dimension adéquate. 


\section{Conclusion}

Les idées développées dans le cadre de ce projet présentent un exemple de ce qu'il est possible de réaliser pour aménager des ouvrages de protection de marinas construites en site vierge, tout en préservant la nature sous tous ses aspects. Une telle démarche faite lors de l'investissement $\mathrm{du}$ projet, doit naturellement s'accompagner d'une démarche similaire permanente au cours de son exploitation. Des précautions doivent être prises an particulier en ce qui concerne la pression anthropique sur les plages et les dunes. 